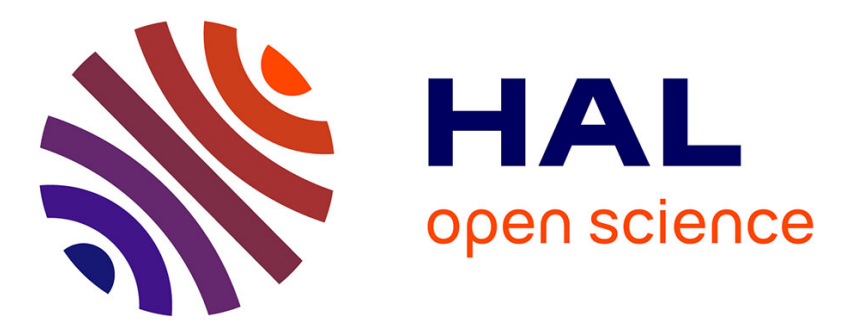

\title{
A Resonant System for In Vitro Studies Emulating Wireless Power Transfer Exposure at $13.56 \mathrm{MHz}$
}

Mohsen Koohestani, Richard Perdriau, Yves Le Dréan, Mauro Ettorre, Maxim Zhadobov

\section{- To cite this version:}

Mohsen Koohestani, Richard Perdriau, Yves Le Dréan, Mauro Ettorre, Maxim Zhadobov. A Resonant System for In Vitro Studies Emulating Wireless Power Transfer Exposure at 13.56 MHz. Bioelectromagnetics, 2020, 41 (5), pp.369-381. 10.1002/bem.22270 . hal-02749675

\section{HAL Id: hal-02749675 \\ https://hal.science/hal-02749675}

Submitted on 9 Nov 2020

HAL is a multi-disciplinary open access archive for the deposit and dissemination of scientific research documents, whether they are published or not. The documents may come from teaching and research institutions in France or abroad, or from public or private research centers.
L'archive ouverte pluridisciplinaire HAL, est destinée au dépôt et à la diffusion de documents scientifiques de niveau recherche, publiés ou non, émanant des établissements d'enseignement et de recherche français ou étrangers, des laboratoires publics ou privés. 


\title{
A Resonant System for In Vitro Studies Emulating Wireless Power
}

\section{Transfer Exposure at $13.56 \mathrm{MHz}$}

\author{
Mohsen Koohestani ${ }^{1,2}$, Richard Perdriau ${ }^{1,2}$, Yves Le Dréan ${ }^{3}$, Mauro Ettorre $^{2}$, Maxim Zhadobov ${ }^{2}$ \\ ${ }^{1}$ Ecole Supérieure d'Électronique de l'Ouest (ESEO), Department of Electrical and Control Engineering, RF-EMC \\ research group, Angers 49107, France \\ ${ }^{2}$ Univ Rennes, CNRS, IETR (Institut d’Électronique et de Télécommunication de Rennes), UMR 6164, F-35000 \\ Rennes, France
}

${ }^{3}$ Univ Rennes, Inserm, EHESP, Irset (Institut de recherche en santé, environnement et travail) - UMR_S 1085, F35000 Rennes, France

Running title: A $13.56 \mathrm{MHz}$ in vitro exposure system

Corresponding author: Mohsen Koohestani, ESEO-IETR, 10 Boulevard Jeanneteau CS 90717, 49107 Angers Cedex 2, France, Tel: +33 (0)2 418667 70, Email: mohsen.koohestani@eseo.fr Conflict of interest: None.

Grant information: French Agency for Food, Environmental and Occupational Health \& Safety (ANSES), under grant nEST-2017/2 RF- 08 (FIGe project), French National Research Agency (ANR) under grant nANR-14-CE26-0030-01 (WIREFREE project), Brittany Region (WiBody project), EU through the European Regional Development Fund (ERDF), Brittany Region, Ministry of Higher Education and Research, Rennes Metropole and Conseil Départemental, through the CPER Project SOPHIE/STIC \& Ondes.

\section{Abstract}


This paper presents the design of a resonant system for in vitro studies to emulate the exposure of a monolayer of cells to a wireless power transfer system operating at $13.56 \mathrm{MHz}$. The design procedure targets a system which maximizes the specific absorption rate (SAR) uniformity on the plane where the layer is cultured, as well as SAR efficiency (defined as SAR over the input power), within the size constraints of a standard incubator. Three resonant wireless power transfer systems with different commonly used loop/coil geometries (cylindrical with circular and square crosssections and annular) were compared to assess the configuration maximizing the considered design criteria. The system performance in terms of reflection and transmission coefficients, as well as generated E- and H-fields, was characterized numerically and experimentally inside the incubator. Moreover, SAR was computed at the monolayer level. The system equipped with cylindrical coils with square cross-sections led to a high EM field uniformity in in vitro biological samples. In particular, the uniformities in $\mathrm{E}$ and SAR at the layer level were within $7.9 \%$ and $5.5 \%$, respectively. This was achieved with the variation in $\mathrm{H}$ below the usually considered $\pm 5 \%$ limit.

Keywords: Exposure system; dosimetry; in vitro; resonant wireless power transfer (WPT); HF measurements.

\section{Introduction}

Resonant wireless power transfer (WPT) technology operating in the high frequency (HF) band has increasingly found its applications in consumer electronics, healthcare, and electric vehicle 
charging [Sample et al., 2011]. There has been an increasing public safety concern related to potential biological effect issues such as the potential health risks due to the electromagnetic field (EMF) radiation of this ubiquitous and indispensable technology [Koohestani et al., 2018]. There exist several international safety guidelines and standards (e.g. ICNIRP, IEEE, and CENELEC) limiting the exposure to EMFs in the frequency range from 0 to $300 \mathrm{GHz}$ [ICNIRP, 1998; ICNIRP, 2018; CENELEC, 2008; IEEE, 2010].

Potential health and biological effects of EMFs have been mainly evaluated through epidemiological, in vitro and in vivo studies. There is a number of controversial results in the evaluation of biological effects due to inaccurate dosimetry and lack of well-characterized exposure systems [Durney et al., 1986; Guy et al., 1999; Kuster et al., 2000; Paffi et al., 2010;]. This fact promoted detailed design and characterization of exposure setups to study potential biological effects, providing design procedure guidelines, optimization, implementation, and validation. In [Kuster et al., 2000], exposure setup guidelines were provided for evaluation, optimization, construction, and verification of EMF exposure characterization. In [Paffi et al., 2010], a practical procedure was proposed for a quality exposure system considering different techniques depending on the reference RF structure (resonant, propagating and radiating) and at different operating frequencies (from $180 \mathrm{MHz}$ up to $50 \mathrm{GHz}$ ).

The uniformity of exposure in in vitro experiments is one of the challenges [Kuster et al., 2000]. A variation of $\pm 3 \%$ up to $\pm 5 \%$ is usually considered acceptable for magnetic $(\mathrm{H})$ field distributions [Zhadobov et al., 2013; Miyakoshi et al., 2008; Sakurai et al., 2012; Mizuno et al., 2014], however, there is no generally accepted rule for electric (E) field and specific absorption rate (SAR). Another concern is the power efficiency of exposure systems. In general, the target is to maximize the EM fields and SAR at the level of cells, while decreasing the required input power. This further explains the reason behind the increasing use of two-coil resonant WPT-emulating systems for in vitro 
experiments in the low $\mathrm{MHz}$ range because of the strong $\mathrm{E}$ - and $\mathrm{H}$-fields around the resonating coils. Note that a one-coil resonant system generates a much lower EM field intensity whereas a higher number of coils is not practical due to size limit imposed by the incubator.

A solenoid is well known to produce strong uniform magnetic fields inside the coil depending on the applied current, geometry, and number of turns per unit length. Several studies investigated the EM exposure to WPT systems with respect to the international safety limits [Mizuno et al., 2014; Mizuno et al., 2015; Chun et al., 2014; Park et al., 2013; Koohestani et al., 2017; Christ et al., 2013]. Among them, in [Mizuno et al., 2014; Mizuno et al., 2015; Chun et al., 2014; Park et al., 2013] cylindrical solenoid-shaped coils have been used as the WPT elements to mimic and assess the WPT exposure in HF band. In [Koohestani et al., 2017; Christ et al., 2013], planar solenoid-shaped coils have been employed. As it is generally unclear which WPT system, in terms of loop/coil geometry and topology, provides a higher uniformity and exposure level of EM fields and specific absorption rate $(\mathrm{SAR})$ with respect to the input power, a comparative study was conducted here to investigate WPT systems of different loops/coils geometries.

Recent in vitro exposure systems have mainly focused on exposure assessment in the extremely low (i.e. tens of Hz) [Yamazaki et al., 2000], very low (i.e. tens of kHz) [Koyama et al., 2014], and high frequencies (i.e. hundreds of MHz) [Zhadobov et al., 2013; Schuderer et al., 2004], while less attention has been paid to the $\mathrm{HF}$ dosimetry at low $\mathrm{MHz}$ frequencies (i.e. from a fraction of $\mathrm{MHz}$ to tens of MHz), increasingly used for WPT technology. There exist several standard operating frequencies (ISM bands) for in vitro HF WPT systems such as $6.78 \mathrm{MHz}$ and/or its harmonics (13.56, or $27.12 \mathrm{MHz}$ ). As a compromise between size constraints imposed by the incubator and achievable transfer efficiency in different frequencies, the mostly used second harmonic (13.56 $\mathrm{MHz}$ ) was adopted in this study. 
There are a few exposure systems designs operating around $13.56 \mathrm{MHz}$, mainly evaluating the exposure to radio frequency identification (RFID) tags and readers (e.g. [Hohbergerm et al., 2009; Seidman et al., 2014]). Conversely, very few in vitro WPT exposure systems operating at 13.56 $\mathrm{MHz}$ are reported in the literature [Mizuno et al., 2014; Mizuno et al., 2015]. In those studies, the position of the tissue culture plate was fixed (between the transmitting and receiving coils closer to the latter), and the coil size was optimized taking only into account the uniformity of the magnetic field distribution in absence of the tissue culture plate and culture medium. Moreover, since such systems are designed to evaluate in vitro biological effects, it is important to cover the power range up to SAR exposure limits. The latter has not been addressed in those studies.

In the current study, we focus on the design and characterization of an experimental setup developed to investigate potential biological effects due to WPT exposure at $13.56 \mathrm{MHz}$. The adequacy of WPT systems with different loop/coil geometries is assessed, considering the uniformity of EM fields and SAR as well as SAR efficiency (defined here as SAR over the input power) as exposure metrics. The general design procedure is different compared to that in [Mizuno et al., 2014; Mizuno et al., 2015]; a $13.56 \mathrm{MHz}$ WPT system with maximum achievable transfer efficiency and impedance matching is designed inside an incubator. The position of the tissue culture plate including the culture medium, which is usually not considered in the exposure system design stage, is selected to maximize the uniformity as well as E-field and SAR with respect to the input power, in addition to the generally considered $\mathrm{H}$-field variation at the bottom of culture medium.

\section{Materials and Methods}

This section presents the design and the simulation strategy of a resonant exposure system using a magnetically coupled WPT operating at $13.56 \mathrm{MHz}$, taking into account the influence of design 
parameters on dosimetric quantities. To avoid repetition, the test bench is introduced first as most of the equipment was modeled in simulations.

\section{A. Exposure Setup and Method}

Figure 1 shows an ad-hoc experimental setup built for in vitro studies at $13.56 \mathrm{MHz}$. The setup comprises two identical resonant coils of 10 turns and $8 \mathrm{~mm}$ pitch, each driven by a single turn loop. Coils are separated by $10 \mathrm{~cm}$ and are axially aligned together with the loops; the distance was chosen to minimize the impact of the incubator as a neighboring conductor on the power transfer characteristics of the system. The loop and coil parameters (i.e. pitch, number of turns, inner/outer radii) were optimized for the systems to resonate at the frequency of interest (i.e. 13.56 $\mathrm{MHz}$ ). The outer dimensions of the loops and coils are $25 \mathrm{~cm} \times 25 \mathrm{~cm}$ and $18.5 \mathrm{~cm} \times 18.5 \mathrm{~cm}$, respectively. They are made of copper rods with $2.5 \mathrm{~mm}$ outer diameter.

The transmitting (Tx) loop is connected via a low-loss and high power cable to the signal generation unit comprising a $100 \mathrm{kHz}-22$ GHz SMD 100A signal generator (Rohde \& Schwarz Munich, Germany) and a 10-15 MHz ref1014-1000 high power amplifier (RFPA, Artigues-prèsBordeaux, France). Depending on the power, the receiving ( $\mathrm{Rx})$ loop is terminated with two different power 50- $\Omega$ loads (Huber \& Suhner 65-N, Herisau, Switzerland, or Bird Technologies Termaline $8251 \mathrm{~N}$, Solon, $\mathrm{OH}$ ). A tissue culture plate made of polystyrene (BD Bioscience, Le Pont De Claix, France), including six wells of $17.5 \mathrm{~mm}$ radius and $20.2 \mathrm{~mm}$ height containing a cell monolayer (from 0.1 to 0.2 million per well) and culture medium, was placed between the coils 10 $\mathrm{mm}(d=10 \mathrm{~mm})$ above the $\mathrm{Rx}$ (for the final system design, this distance might be different; see more details in Section III). The culture medium (6 ml per well, almost half filled) is Dulbecco's modified Eagle medium (DMEM, Gibco/Life Technologies, Carlsbad, CA) supplemented with 8\% fetal calf serum (FCS), 1\% antibiotics, and 1\% L-glutamine. The dielectric properties of the culture medium at the frequency of interest were measured using a $10 \mathrm{MHz}-3 \mathrm{GHz}$ DAK-12 coaxial 
dielectric probe kit (SPEAG, Zurich, Switzerland) at $37 \pm 0.2{ }^{\circ} \mathrm{C}$ and are shown in Figure 2. The relative permittivity and conductivity of the DMEM medium at $13.56 \mathrm{MHz}$ are 120.2 and 1.375 $\mathrm{S} / \mathrm{m}$, respectively.

A Holaday (ETS-Lindgren, Cedar Park, TX) HI-2200 radio frequency (RF) meter equipped with a $\mathrm{H} 210$ magnetic field probe $(300 \mathrm{kHz}-30 \mathrm{MHz}, 0.3$ to $30 \mathrm{~A} / \mathrm{m}$ dynamic range) and E100 electric field probe (1 MHz-4 GHz, 0.3 to $800 \mathrm{~V} / \mathrm{m}$ dynamic range) were used for EM field measurements. For an increased accuracy, since the averaging volume and influence of the probes can be considered significant, the probes were modeled in simulations.

The system was located inside an IFE 400 incubator (Memmert, Büchenbach, Germany) with inner dimensions $400 \times 330 \times 400 \mathrm{~mm}^{3}$ to maintain the temperature at $37{ }^{\circ} \mathrm{C}$. A stand made of Plexiglas $\varepsilon_{\mathrm{r}}=3.4$ and $\tan \delta=0.001$ at $13.56 \mathrm{MHz}$ ) was designed to hold the WPT system elements and tissue culture plate (Fig. 1).

It is worth stating that the system is equipped with a carrier for the optical fiber probes that can be used for temperature measurements. Heating characterization is beyond the scope of this initial study, which is focused on low-power bioelectromagnetic experiments.

\section{B. Simulation Setup and Method}

The test bench was entirely reproduced in simulations, in terms of geometry and dimensions, excluding the input/output cables. Ansys HFSS commercial full-wave electromagnetic simulation package based on finite element method [HFSS, 2018] was used for numerical analysis. In the numerical models, the WPT elements (loops and coils) and the metallic parts of the incubator were considered copper and stainless steel, respectively. Polystyrene $\left(\varepsilon_{\mathrm{r}}=2.6\right.$ and $\tan \delta=0.001$ at 13.56 MHz) was chosen for the culture plate. The measured electromagnetic properties of the culture medium with $1.16 \mathrm{~g} / \mathrm{cm}^{3}$ mass density were set in simulations (see more details in the next 
section). The materials used to model the field probes were plastic and FR4 epoxy for the sensor protection cover and support, respectively. 50- $\Omega$ coaxial connectors were only modeled for the input and output ports of the final system design.

Simulations were performed using a rigorous iterative algorithm based on mixed order basis functions to refine the mesh, noting that mixed order efficiency is comparable or better than all single order basis functions in terms of total mesh elements, number of convergence passes, elapsed time and memory [HFSS, 2018]. This was confirmed in the first simulation to study the WPT system performance, and then considered for the rest of the simulations. A local mesh of 0.1-mm resolution was used in simulations at the bottom of each well. The considered dosimetric quantities are $\mathrm{E}, \mathrm{H}$, and local SAR, and all presented results were computed for $1 \mathrm{~W}$ input power at the system resonant frequency, i.e. $13.56 \mathrm{MHz}$. Note that the proposed in vitro system was designed for exposure conditions when the cells are attached to the bottom of a well. Therefore, due to the tiny cell's thickness (typically 5-15 $\mathrm{\mu m}$ ), a local (unaveraged) rather than averaged SAR was considered. Moreover, due to the negligible impact of the cell monolayer on EM field and SAR distributions within a well [Zhadobov et al., 2013], due to its very tiny thickness, it was not included in the numerical model.

Since it was practically impossible to measure the EM fields in the wells with the field probes larger than the wells' dimensions, an indirect approach was used to quantify the EM field in the tissue culture medium. To this aim, we first numerically simulated the designed WPT system inside the incubator in presence of the tissue culture plate containing the samples to obtain the EM field distributions in the incubator. Both E- and H-fields inside the incubator were then measured and compared to the numerical data to validate the simulations; a similar approach was previously employed in [Zhadobov et al., 2013]. Finally, the EM field and SAR distributions inside the tissue culture medium were analyzed numerically. 


\section{Impact of different parameters on dosimetric quantities}

Three exposure systems with different coil geometries (cylindrical with circular and square crosssections, and annular) operating at $13.56 \mathrm{MHz}$ were designed, targeting a maximum power transfer efficiency (defined as the square of the transmission coefficient $\left|S_{21}\right|^{2}$ [Koohestani et al., 2017]) inside the incubator (Fig. 3). The latter is important to ensure optimal coupling among WPT elements, which further provides higher EM field intensity [Mizuno et al., 2014; Koohestani et al., 2017]. As described in subsection II-A, each configuration consists of two identical resonant coils, each one driven by a single turn loop, which were optimized according to the methodology suggested in [Koohestani et al., 2017] for a fixed distance of $10 \mathrm{~cm}$ between Tx and Rx coils; detailed design parameters are given in Table I. The three considered WPT systems have an input matching level better than $-20 \mathrm{~dB}$ (0.01 in linear) with a maximized transfer efficiency greater than $90 \%$ at the frequency of interest.

For the next step, the tissue culture plate was moved along the coil axis with $d$ ranging from -20 $\mathrm{mm}$ to $+30 \mathrm{~mm}$ with a $10 \mathrm{~mm}$ step to find its optimum position for the highest possible uniformity and level of EM fields and SAR; $d=0 \mathrm{~mm}$ refers to the lower side of the culture plate being in line with the upper plane of the Rx coil, as shown in Figure 3c, and the minimum of $d=-20 \mathrm{~mm}$ was chosen to ease the access to the tissue culture plate during the exposure experiments. Note that the wells of the tissue culture plate were fully filled with the culture medium.

Moreover, depending on the incubator and the WPT system dimensions, the tissue culture plate orientation can be important as it may help biologists to reach the plate easier. To further investigate the impact of orientation, for a fixed value of $d=+10 \mathrm{~mm}$, the tissue culture plate was rotated by $90^{\circ}$. 
Furthermore, one advantage of the designed WPT system is to provide the highest possible matching level and efficiency without using matching circuits connected to the system. However, it is common to facilitate the integration of an external matching circuit to adjust the system operating frequency whatever the design, which is indeed more practical. This is known to reduce the coupling between the WPT system elements in free space, and consequently lower fields and exposure levels [Koohestani et al., 2017]. The aim is to assess to what extent that reduction in the field strength influences the level, variation, and uniformity of E, H, and SAR at the bottom of the tissue culture dish inside the incubator. As a demonstration showcase, although it may not be

practical, to achieve that goal, the length of the Tx coil was initially increased (by $8.9 \mathrm{~mm}$ ) to be able to shift down the system resonant frequency on purpose (to $13 \mathrm{MHz}$ ). The latter was then shifted up from 13 to $13.56 \mathrm{MHz}$ using additional LC matching circuits connected to its two ports $(\mathrm{L}=1 \mu \mathrm{H}$ in series, $\mathrm{C}=270 \mathrm{pF}$ in parallel).

Finally, the culture medium volume is usually chosen between a half of and almost full well. It is mainly needed to provide the nutrition to cells, and to avoid possible leakage to the neighboring wells by touching the culture plate cover during transportation. Since the volume of the culture medium may vary for different sets of in vitro experiments, it may potentially impact the SAR distribution and total power absorption in the culture medium, as well as resulting heating. Therefore, we investigate the impact of the culture medium volume, being half- or fully-filled, on the uniformity and exposure level of EM field and SAR.

\section{Numerical Results}

This section deals with the numerical results obtained following the methodology to design a resonant exposure system at $13.56 \mathrm{MHz}$. First, a comparison study among the most commonly used geometries for WPT loops/coils was performed to assess the configuration providing (i) the most uniform E, H, and SAR distributions and (ii) the highest SAR efficiency. It was then followed 
by a parametric study to find the optimal location as well as the impact of the orientation of the tissue culture plate between the Tx and Rx parts, in accordance with the two previously mentioned design criteria. Finally, the influence of key parameters contributing to the results was assessed such as the use of a matching network to adapt the system at a desired frequency, and the volume of the culture medium inside the wells. Variation, also known as coefficient of variation, of the considered dosimetric quantities was calculated as the ratio of standard deviation to mean value [Kuster et al., 2000]; it is usually taken as the non-uniformity degree of dosimetric quantities within one well. Note that the mean value and variation were evaluated from the monitored $\mathrm{E}, \mathrm{H}$, and SAR over the 2D circular surface at the bottom of the wells (where the cell monolayer is located) extracted from HFSS. In all tables, E, H and SAR are denoted "mean \pm standard deviation". Moreover, uniformity was calculated as the difference among variations in the wells.

Furthermore, since $1 \mathrm{~W}$ input power was considered in all simulations, SAR efficiency is numerically equal to SAR, but expressed in $\mathrm{kg}^{-1}$. Only SAR efficiency was considered, as SAR is a function of $\mathrm{E}$ and only variation (not levels) matters for $\mathrm{H}$.

\section{A. WPT Coil Geometry}

Table II presents the magnitude and variation of E, H, and SAR obtained at the bottom of only wells $\# 1$ and $\# 2$ as showcases $^{1}$ with the tissue culture plate placed $10 \mathrm{~mm}$ above the $\mathrm{Rx}$ coil. While all the considered exposure systems result in almost similar E, H, and SAR variations, the square loops/coils configuration provides higher exposure levels compared to the other two geometries; the SAR efficiency of the cylindrical/square WPT was increased by a maximum of $30.7 \%$ and $423 \%$ with respect to the cylindrical/circular and annular systems, respectively. In other words, using a WPT system with square loops/coils makes it possible to obtain a better SAR

\footnotetext{
${ }^{1}$ mainly for the sake of brevity. Results for all six wells are only provided in section IV for the final design of the system.
} 
efficiency, with only a small impact on uniformity. This leads to system cost reduction by considering a power amplifier with much lower output power. Hence, for the rest of the study, a WPT system equipped with square loops/coils was considered.

\section{B. Impact of Tissue Culture Plate}

(1) Position: A Plexiglas stand for the WPT system was included in the simulations. It is worth mentioning that comparing the results provided in Table II and Table III for a square WPT with $d=+10$ mm clearly shows the impact on dosimetric quantities; the SAR efficiency decreases by $5.9 \%$ in well $\# 1$ while being unchanged in well $\# 2$. Moreover, the mismatch due to the tiny frequency shift ( $<0.02 \mathrm{MHz}$, verified with measurements) caused by the movement of the culture plate was compensated by adjusting the input power.

E, H, and SAR variations and levels are summarized in Table III. As it can be seen, with the increase of distance $d$, the exposure level decreases while the variation gradually increases at distances larger than $d=+10 \mathrm{~mm}$. Although the magnetic field variation was below $\pm 5 \%, \mathrm{SAR}$ efficiency results for $d=-20 \mathrm{~mm}$ and $+10 \mathrm{~mm}$ were compared in order to monitor the highest possible impact of the culture plate position, ignoring cases with $d=+20 \mathrm{~mm}$ and $+30 \mathrm{~mm}$, due to the inhomogeneity of SAR in wells $\# 1$ and $\# 2$. With the increase of $d$ from -20 to $+10 \mathrm{~mm}$, SAR efficiency decreases by $31.9 \%$ and $39.1 \%$ in wells $\# 1$ and $\# 2$, respectively, while the uniformity varies by a minimum of $3.3 \%$ (between -20 and $-10 \mathrm{~mm}$ in well $\# 2$ ) to a maximum of $18.4 \%$ (between 0 and $+10 \mathrm{~mm}$ in well $\# 1$ ).

Since the considered $13.56 \mathrm{MHz}$ frequency is above the frequencies where the nerve stimulation effects are dominant, only SAR was considered in the study [ICNIRP, 1998; ICNIRP, 2018]. To mimic the worst case scenario, the SAR limit of $2 \mathrm{~W} / \mathrm{kg}$ for head and trunk (even if it refers to an averaged SAR) was considered instead of the one for limbs $(4 \mathrm{~W} / \mathrm{kg})$, as a reference for laboratory 
intercomparison on biological data. In order to provide an estimation of the maximum allowable input power (MAIP), its value satisfying the aforementioned SAR limit was calculated. To not exceed the SAR limit, with the dosimetric values in well $\# 1$, the MAIP for $d=-20 \mathrm{~mm}$ and +10 $\mathrm{mm}$ were $21.2 \mathrm{~W}$ and $31.2 \mathrm{~W}$, respectively. For power and associated cost concerns, $d=-20 \mathrm{~mm}$ can be considered a suitable position, as it offers a good trade-off between the highest possible SAR efficiency and uniformity.

(2) Orientation: Table IV provides E, H, and SAR at wells $\# 1$ and $\# 2$ for $1 \mathrm{~W}$ input power at 13.56 $\mathrm{MHz}$ for a tissue culture plate rotated by $90^{\circ}$. Comparing the results in Table III for $d=$ $+10 \mathrm{~mm}$ to those given in Table IV indicate higher $\mathrm{E}, \mathrm{H}$, and SAR levels with different impacts on variation. The SAR efficiency in wells \#1 and \#2 for the considered orientations were increased by $5.9 \%$ and $6.9 \%$, respectively. Therefore, we conclude that changing the orientation of the tissue culture plate can help to reduce cost and to increase the SAR efficiency at the expense of uniformity.

\section{Impact of Tuning Circuit}

Impact of matching circuit, connected to the WPT system to detune its resonance from 13 to 13.56 $\mathrm{MHz}$, on $\mathrm{E}, \mathrm{H}$, and $\mathrm{SAR}$ are given in Table $\mathrm{V}$ for wells $\# 1$ and $\# 2$. They indicate a dramatic decrease in uniformity and the exposure levels when a matching circuit was used for compensation, compared to the design with $13.56 \mathrm{MHz}$ "self-resonant" system; SAR efficiency was reduced by $75 \%$ and $79.3 \%$ in wells $\# 1$ and $\# 2$, respectively). This in return demands for an increased power and budget; for example, the MAIP satisfying the ICNIRP limit in terms of SAR is of the order of tens of watts $(42.5 \mathrm{~W})$ with the "self-resonant" design, whereas it significantly increases to hundreds of watts $(206.1 \mathrm{~W})$ when connected to a matching circuit. Note that the lower dosimetric quantities at $13 \mathrm{MHz}$ compared to those in Table III for a "self-resonant" system with similar d = 
-20 $\mathrm{mm}$ but operating at $13.56 \mathrm{MHz}$ are due to modifying only the Tx coil and, therefore, reduced optimal coupling between the system elements.

\section{Impact of Culture Medium Volume}

E, H, and SAR at wells \#1 and \#2 for $1 \mathrm{~W}$ input power at $13.56 \mathrm{MHz}$ with a half-filled tissue culture plate medium are provided in Table VI. Comparing the results in wells \#1 and \#2 when being half- or fully-filled (the latter provided in Table III for $d=-20 \mathrm{~mm}$ ) show a tiny influence of the culture medium volume; SAR efficiency decreases by $1 \%$ in well \#1 while being unchanged in well $\# 2$. Of course, the results may vary depending on the surface and size of the culture plates.

\section{Experimental Results}

Figure 1 shows the fabricated system. Here, we experimentally validate the proposed exposure system design for in vitro studies. Due to the impracticality of EM field and SAR measurements directly at the bottom of the wells, the performance of the system is measured inside an incubator and compared to that obtained numerically in terms of input reflection and transmission coefficients as well as E- and H-field distributions. Moreover, the EM field and SAR inside the culture medium are computed. All measurements were performed in presence of the tissue culture plate containing half-filled DMEM culture medium, with $d=-20 \mathrm{~mm}$.

\section{A. Input Reflection and Transmission Coefficients Inside Incubator}

An Agilent FieldFox RF analyzer N9912A (2 MHz-6 GHz) calibrated with the Keysight 85515A 4-in-1 module at the end of the input/output cables, was used to monitor scattering parameters. Figure 4 plots the simulated and measured $\left|S_{11}\right|$ and $\left|S_{21}\right|$ of the system inside the incubator. As observed, the WPT system sharply resonates at the central frequency of $13.56 \mathrm{MHz}$. Measurements 
closely follow simulations, which is expected as most of the details in experimental setup were implemented in simulations. According to the experiments:

- a $1.4 \mathrm{~dB}$ (1.17 times) difference in simulated and measured $\left|\mathrm{S}_{21}\right|$ can be observed

- the tissue culture plate led to a negligible frequency shift $(<0.01 \mathrm{MHz})$

- the shift of the tissue culture plate position using an additional Plexiglas spacer was found to cause a tiny frequency shift $(<0.02 \mathrm{MHz})$.

Since the calibration was performed at the end of the cables, the difference in simulated and measured $\left|\mathrm{S}_{21}\right|$ may not be attributed to the power losses associated with the cables connected to Tx and Rx. Instead, it can be due either to the WPT elements positioning inaccuracies or the absence of those cables in simulations which can slightly modify the field distributions inside the incubator. Moreover, the negligible frequency shift due to the culture plate position was numerically predicted, and is related to the fact that, in short-range WPT systems, $\mathrm{H}$ is dominant compared to E, and hence no disturbances due to the presence of non-magnetic materials occur.

\section{B. EM Field Inside the Incubator}

Measurements were conducted to monitor the EM field magnitudes and distributions inside the incubator. The door of the incubator was replaced by a 2-cm-thick polyvinyl chloride dielectric plate, covered by a 0.05-mm-thick adhesive copper tape, as can be seen in Figure 5 . Nine holes with a diameter similar to the E- and $\mathrm{H}$-field probes were created in the plate to allow positioning the probes inside the incubator; for the sake of readability, only five positions were numbered in the HFSS model in Figure 5, however, the model and the photograph in the same figure clearly show nine holes corresponding to the measurement positions. A first measurement confirmed the insensitivity of the input reflection coefficient of the WPT system to the presence of the probes at different hole locations. 
For the sake of brevity, in Figure 5 the results are shown only for holes number \#4 and \#5 (the inserted picture with the field probe is just an example of experimental setup for hole \#2), being in the area of interest where the tissue culture plate is present (hole $\# 5$ passes across the coil axis in the center whereas hole $\# 4$ is positioned $3 \mathrm{~cm}$ off the center in $y$ direction). It is worth mentioning that:

- due to the dynamic range limit of $\mathrm{E}$ and $\mathrm{H}$ probes, measurements were performed for an input power of $+10 \mathrm{dBm}(10 \mathrm{~mW})$. Results were then rescaled to an input power of $1 \mathrm{~W}$ $(+30 \mathrm{dBm})$ and compared to simulations

- the field probes were moved toward the center $y$-line of the incubator by $10 \mathrm{~mm}$ steps; the assessable distance was limited due to probe length

A fairly good agreement is observed between simulations and measurements. The E-field deviation around $d=150 \mathrm{~mm}$ can be due to dielectric objects (electronics and cables used inside the probe structure) that disturbed the field measurements; a similar behavior is noted in a WPT field experiment in free space [Koohestani et al., 2017]. In order to demonstrate the WPT operation inside the incubator, Figure 6 exhibits the EM field distributions around the WPT elements in three orthogonal planes passing by the center of the $\mathrm{Tx} / \mathrm{Rx}$ for $1 \mathrm{~W}$ input power at $13.56 \mathrm{MHz}$. The EM field maxima are mainly distributed around the resonant coils, being more pronounced near the coil windings and at the coil center axis.

\section{EM Field and SAR Inside the Culture Medium}

Figure 7 shows the computed E, H, and SAR distributions at the bottom of the tissue culture plate for an input power of $1 \mathrm{~W}$ at $13.56 \mathrm{MHz}$, for the configurations presented in Fig. 6. A first look at the results indicates the identical E and SAR distributions and exposure values in the wells, which is related to uniformity of the E-field where the tissue culture plate was located. 
Considering the $\mathrm{H}$ scale, the distributions in the wells can also be considered almost uniform, especially in wells \#2 and \#5. Table VII presents the variation and exposure levels of the considered dosimetric quantities. In line with the E and SAR distributions shown in Figure 7, the uniformities in $\mathrm{E}$ and SAR are limited among the wells to a maximum of $7.9 \%$ and $5.5 \%$, respectively, considered suitable for experimental studies in the absence of additional criteria in the current literature at these frequencies. Meanwhile, although being below the generally considered $\pm 5 \%$ limit, a higher $\mathrm{H}$ variation is observed in wells $\# 2$ and \#5. Notice that the lower exposure values compared to those in Table III for similar $d=-20 \mathrm{~mm}$ is due to the inclusion of the Plexiglas support for the tissue culture plate in the numerical analysis; the Plexiglas support was previously found to reduce the SAR efficiency (section numerical results, subsection B).

It is also worth mentioning that an optimization without the culture plate and medium would lead to very different results. To further confirm that assumption, another simulation was carried out in those conditions, resulting in an E-field about 100 times higher than with the plate and medium, with higher variation (e.g. in well \#1,30.64\% instead of $23.18 \%$ ).

For an estimation of the MAIP, the latter was computed to verify the compliance with ICNIRP SAR limit. Table VIII summarizes the MAIP to satisfy local SAR ICNIRP basic restrictions at 13.56 MHz for the final configuration of the exposure system. As observed, the MAIP that satisfies the SAR limit varies significantly by a maximum of $21.9 \%$ among all wells, even though there are minor SAR efficiency differences in the obtained values in wells $\# 1, \# 3$, $\# 4$ and $\# 6$.

\section{Conclusion}

The design procedure of a $13.56 \mathrm{MHz}$ resonant exposure system for in vitro experiments is presented, aiming at a system which provides maximum possible uniformity and SAR efficiency in the culture medium containing cells, within the size constraints imposed by the incubator. Three 
resonant wireless power transfer systems with different commonly used loop/coil geometries (cylindrical with circular and square cross-sections, and annular) were compared to assess the one maximizing the considered design criteria. It was followed by a parametric study to find the optimal tissue culture plate location within the system. Simulations and experiments were performed inside the incubator in terms of reflection and transmission coefficients as well as Eand H-field distributions.

By including most experimental details in the numerical model, a fairly good agreement was achieved between the simulated and measured reflection and transmission coefficients, as well as for the electric and magnetic fields. The system equipped with cylindrical coils with square crosssections led to a high EM field uniformity in the in vitro biological samples. The design procedure was verified by obtaining a $\mathrm{H}$ variation below the usually considered limit $( \pm 5 \%)$. Meanwhile, the uniformities in E and SAR were limited among the wells at the level of cells to a maximum of $7.9 \%$ and $5.5 \%$, respectively. The system is currently used in in vitro studies to investigate cellular stress.

\section{Acknowledgement}

The authors would like to cordially thank L. Cronier, C. Guitton, and X. Morvan for their help during the fabrication of the system.

\section{References}

Ansys Inc, High Frequency Structure Simulator (HFSS). 2018. Electromagnetics Suite v19.1, Pittsburgh (PA), USA. 
CENELEC EN 50413. 2008. Basic standard on measurement and calculation procedures for human exposure to electric, magnetic and electromagnetic fields $(0 \mathrm{~Hz}-300 \mathrm{GHz})$.

Christ A, Douglas MG, Roman JM, Cooper EB, Sample AP, Waters BH, Smith JR, Kuster N. 2013. Evaluation of wireless resonant power transfer systems with human electromagnetic exposure limits. IEEE Trans Electromag Compatibility 55:265-274.

Chun Y, Park S, Kim J, Kim Ji, Kim H, Kim Jo, Kim N, Ahn S. 2014. Electromagnetic compatibility of resonance coupling wireless power transfer in on-line electric vehicle system. IEICE Trans Communications E97B:416-423.

Durney CH, Massoudi H, Iskander MF. 1986. Radiofrequency Radiation Dosimetry Handbook, 4th edition Brooks AFB, TX: USAF SAM, 1986, Rep. USAFSAM-TR-85-73.

Guy AW, Chou CK, McDougall JA. 1999. A quarter century of in vitro research: A new look at exposure methods. Bioelectromagnetics 20:21-39.

Hohbergerm CP, Tsirline BY. 2009. Design of a $13.56 \mathrm{MHz}$ segmented Helmholtz coil for RF exposure testing of biologics to simulated RFID readers. Int J Radio Freq Identification Technol Applications 2:65-92.

ICNIRP 1998: Guidelines for limiting exposure to time-varying electric, magnetic, and electromagnetic fields (up to $300 \mathrm{GHz}$ ). Health Phys. 74:494-522.

ICNIRP 2018: Guideline for limiting exposure to time-varying electric, magnetic fields (100 kHz to $300 \mathrm{GHz}$ ). 2018. Draft ICNIRP Guidelines.

IEEE Standard for safety levels with respect to human exposure to radio frequency electromagnetic fields, $3 \mathrm{kHz}$ to $300 \mathrm{GHz}$. 2010. ISBN 978-0-7381-6207-2 STD96039. 
Koohestani M, Zhadobov M, Ettorre M. 2017. Design methodology of a printed WPT system for HF-band mid-range applications considering human safety regulations. IEEE Trans Microw Theory Techn 65:270-279.

Koohestani M, Zhadobov M, Ettorre M. 2018. Local dosimetry applied to wireless power transfer around $10 \mathrm{MHz}$ : dependence on EM parameters and tissue morphology. IEEE Journal of Electromag RF and Microw in Medicine and Biology 2:123-130.

Koyama Sh, Narita E, Shinohara N, Miyakoshi J. 2014. Effect of an Intermediate-Frequency Magnetic Field of $23 \mathrm{kHz}$ at $2 \mathrm{mT}$ on Chemotaxis and Phagocytosis in Neutrophil-Like Differentiated Human HL-60 Cells. Int J Environ Res Public Health 11:9649-9659.

Kuster N, Schonborn F. 2000. Recommended minimal requirements and development guidelines for exposure setups of bio-experiments addressing the health risk concern of wireless communications. Bioelectromagnetics 21:508-514.

Miyakoshi J, Horiuchi E, Nakahara T, Sakurai T. 2008. Magnetic fields generated by an induction heating (IH) cooktop do not cause genotoxicity in vitro. Bioelectromagnetics 28:529-537.

Mizuno K, Miyakoshi J, Shinohara N. 2014. In vitro exposure system using magnetic resonant coupling wireless power transfer. Wireless Power Transfer 1:97-107.

Mizuno K, Shinohara N, Miyakoshi J. 2015. In vitro evaluation of genotoxic effects under magnetic resonant coupling wireless power transfer. Int J Environ Res Public Health 12:3853-3863.

Paffi A, Apollonio F, Lovisolo GA, Marino C, Pinto R, Repacholi M, Liberti M. 2010. Considerations for developing an RF exposure system: a review for in vitro biological experiments. IEEE Trans Microw Theory Techn 58:2702-2715. 
Park SW, Wake K, Watanabe S. 2013. Incident electric field effect and numerical dosimetry for a wireless power transfer system using magnetically coupled resonances. IEEE Trans Microw Theory Techn 61:3461-3469.

Sakurai T, Narita E, Shinohara N, Miyakoshi J. 2012. Intermediate frequency magnetic field at 23 $\mathrm{kHz}$ does not modify gene expression in human fetus-derived astroglia cells. Bioelectromagnetics $33: 662-669$.

Sample AP, Meyer DT, Smith JR. 2011. Analysis, experimental results, range adaptation of magnetically coupled resonators for wireless power transfer. IEEE Trans Industrial Electronics $58: 544-554$.

Schuderer J, Spat D, Samaras T, Oesch W, Kuster N. 2004. In vitro exposure systems for RF exposures at 900 MHz. IEEE Trans Microw Theory Techniques 52:2067-2075.

Seidman SJ, Bekdash O, Gaug J, Mehryar M, Booth P, Frisch P. 2014. Feasibility results of an electromagnetic compatibility test protocol to evaluate medical devices to radio frequency identification exposure. BioMedical Engineering OnLine 13:1-8.

Yamazaki K, Fujinami H, Shigemitsu T, Nishimura I. 2000. Low stray ELF magnetic field exposure system for in vitro study. Bioelectromagnetics 21:75-83.

Zhadobov M, Ferrand G, Loung M, Soubere Y, Le Couement C, Carton PH, Piret Y, Sauleau R, Le Drean Y. 2013. Exposure system and dosimetry for in vitro studies of biocompatibility of pulsemodulated RF signals of ultrahigh field MRI. IEEE Trans Biomed Engineering 60:3167-3175. 


\section{Figure Caption}

Fig. 1. Schematic representation of the exposure system at $13.56 \mathrm{MHz}$ inside an incubator.

Fig. 2. Measured dielectric properties of the used culture medium at $37{ }^{\circ} \mathrm{C}$.

Fig. 3. Exposure systems of different loops/coils geometries used for the purpose of this study: (a) cylindrical with circular cross-section, (b) annular, (c) cylindrical with square cross-section.

Fig. 4. Scattering parameters of the proposed WPT system inside the incubator.

Fig. 5. Simulated and measured $\mathrm{E}$ - and $\mathrm{H}$-field distributions at $13.56 \mathrm{MHz}$ along a line passing through holes created on the incubator door: (a) E, hole \#4; (b) E, hole \#5; (c) H, hole \#4; (d) H, hole \#5.

Fig. 6. E- and H-field distributions (in $\log$ scale) inside the incubator at planes passing by the center of the $\mathrm{Tx} / \mathrm{Rx}$ for an input power of $1 \mathrm{~W}$ at $13.56 \mathrm{MHz}$.

Fig. 7. $\mathrm{E}, \mathrm{H}$, and SAR distributions at the bottom of the culture medium for an input power of 1 W at $13.56 \mathrm{MHz}$. 


\section{Table Caption}

Table I: Design parameters of $13.56 \mathrm{MHz}$ WPT system elements with different geometries

Table II: $\mathrm{E}, \mathrm{H}$, and SAR at wells \#1 and \#2 for different configurations of WPT systems with $d$ $=10 \mathrm{~mm}$ and $1 \mathrm{~W}$ input power at $13.56 \mathrm{MHz}$

Table III: $\mathrm{E}, \mathrm{H}$, and SAR at wells \#1 and \#2 for the WPT system for different $d$ and $1 \mathrm{~W}$ input power at $13.56 \mathrm{MHz}$

Table IV: $\mathrm{E}, \mathrm{H}$, and SAR at wells $\# 1$ and $\# 2$ for $1 \mathrm{~W}$ input power at $13.56 \mathrm{MHz}$ for a tissue culture plate rotated by $90^{\circ}$

Table V: Impact of matching circuit, connected to the WPT system to shift its resonance from 13 to $13.56 \mathrm{MHz}$, on $\mathrm{E}, \mathrm{H}$, and $\mathrm{SAR}$ at wells $\# 1$ and $\# 2$ for $1 \mathrm{~W}$ input power

Table VI: E, H, and SAR at wells $\# 1$ and $\# 2$ for $1 \mathrm{~W}$ input power at $13.56 \mathrm{MHz}$ with a half-filled tissue culture plate medium

Table VII: E, H, and SAR for the final configuration of the exposure system with $1 \mathrm{~W}$ input power at $13.56 \mathrm{MHz}$

Table VIII: MAIP to satisfy local SAR at $13.56 \mathrm{MHz}$ for the final configuration of the exposure system 


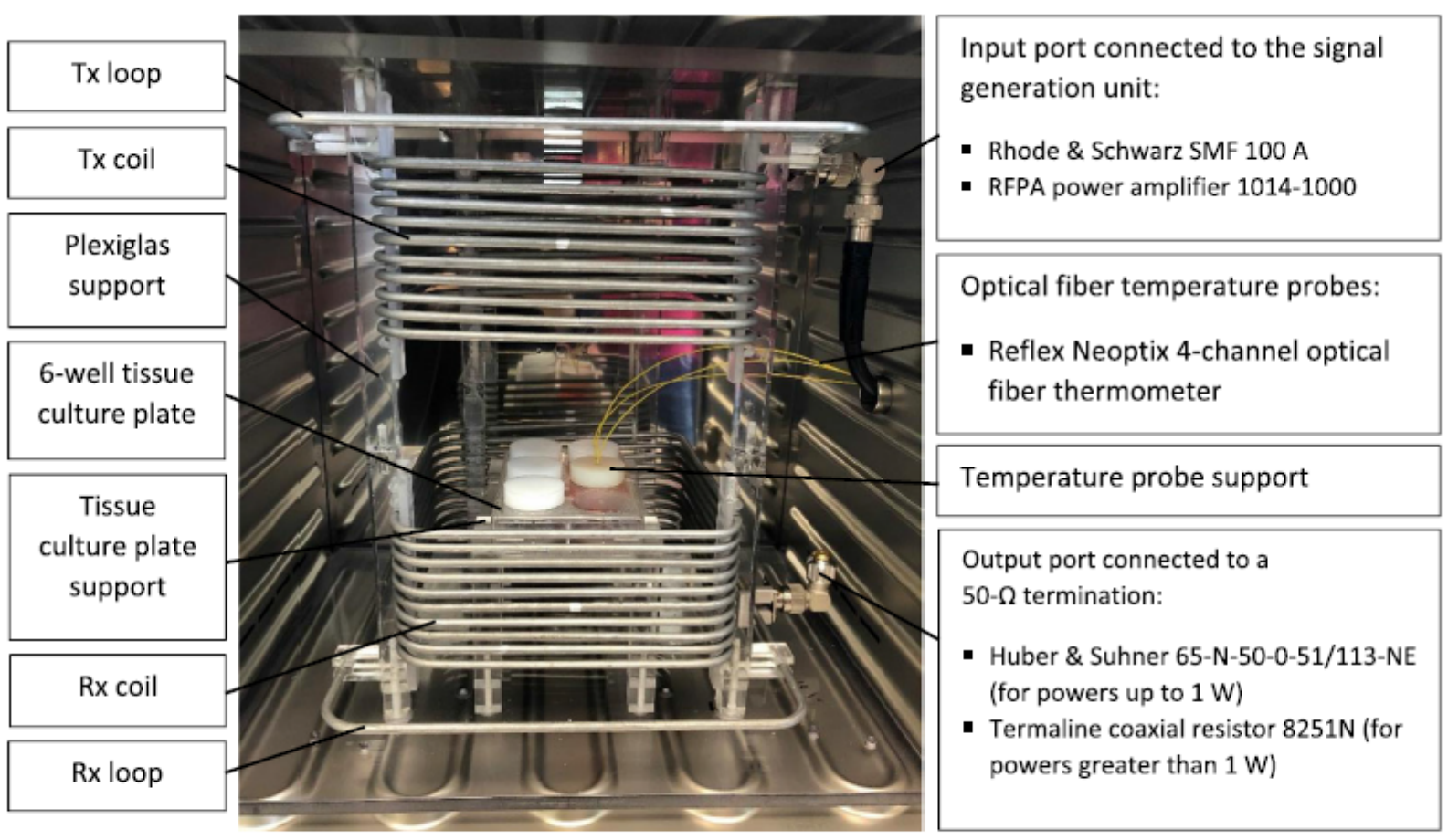

Fig. 1 Schematic representation of the exposure system at $13.56 \mathrm{MHz}$ inside an incubator.

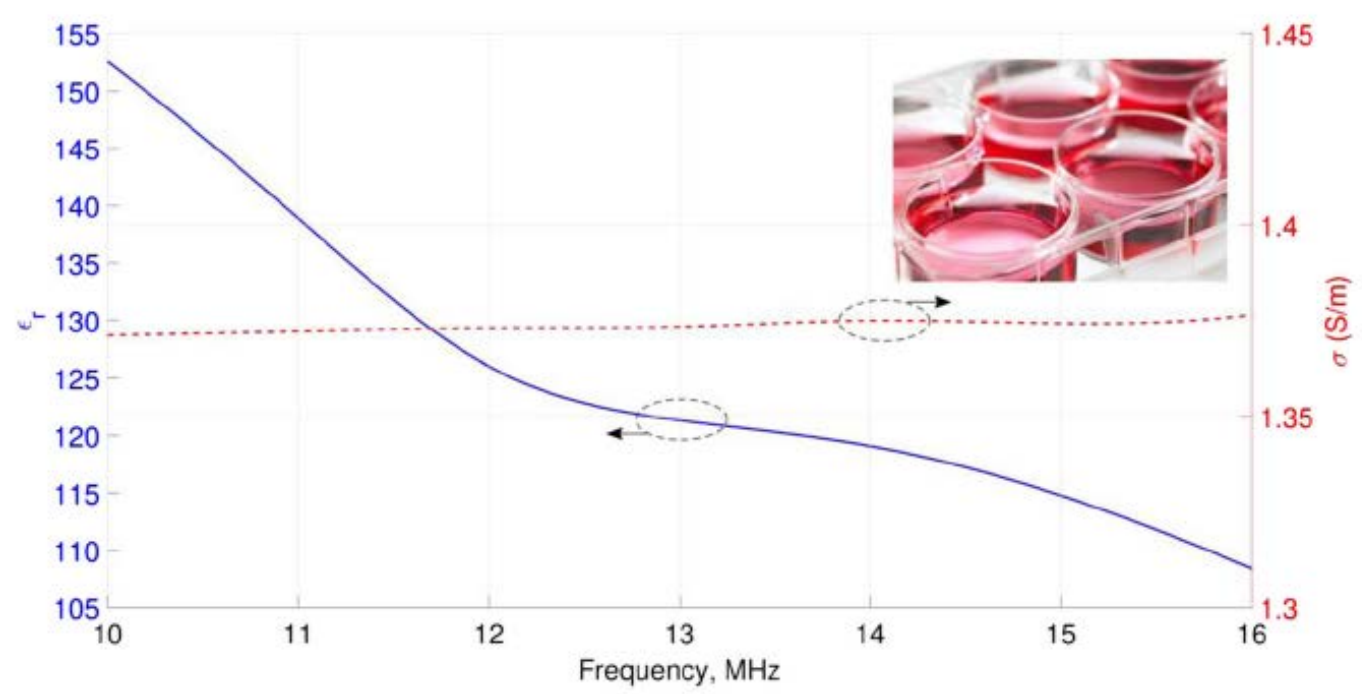

Fig. 2 Measured dielectric properties of the used culture medium at $37^{\circ} \mathrm{C}$. 
(a)

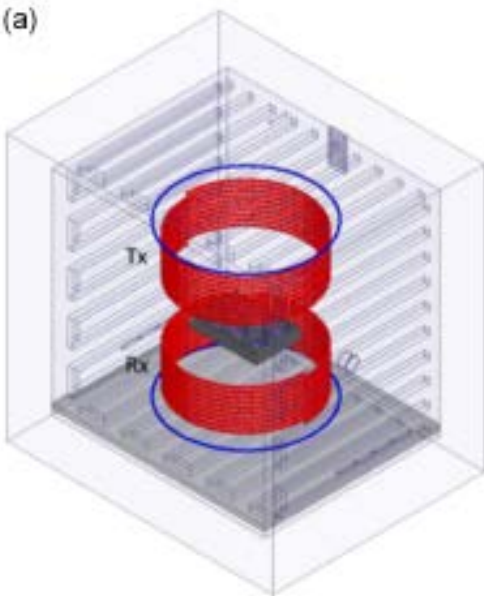

(b)

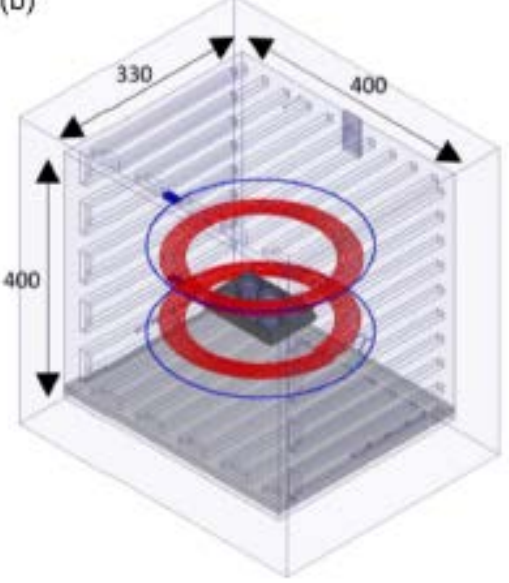

(c)

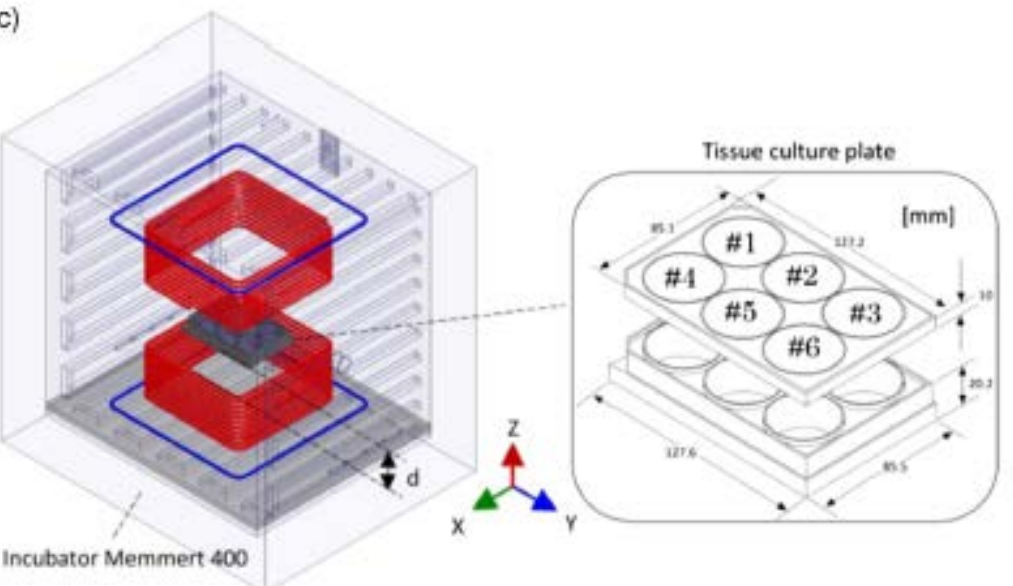

Fig. 3 Exposure systems of different loops/coils geometries used for the purpose of this study: (a) cylindrical with circular cross-section, (b) annular, (c) cylindrical with square cross-section. 


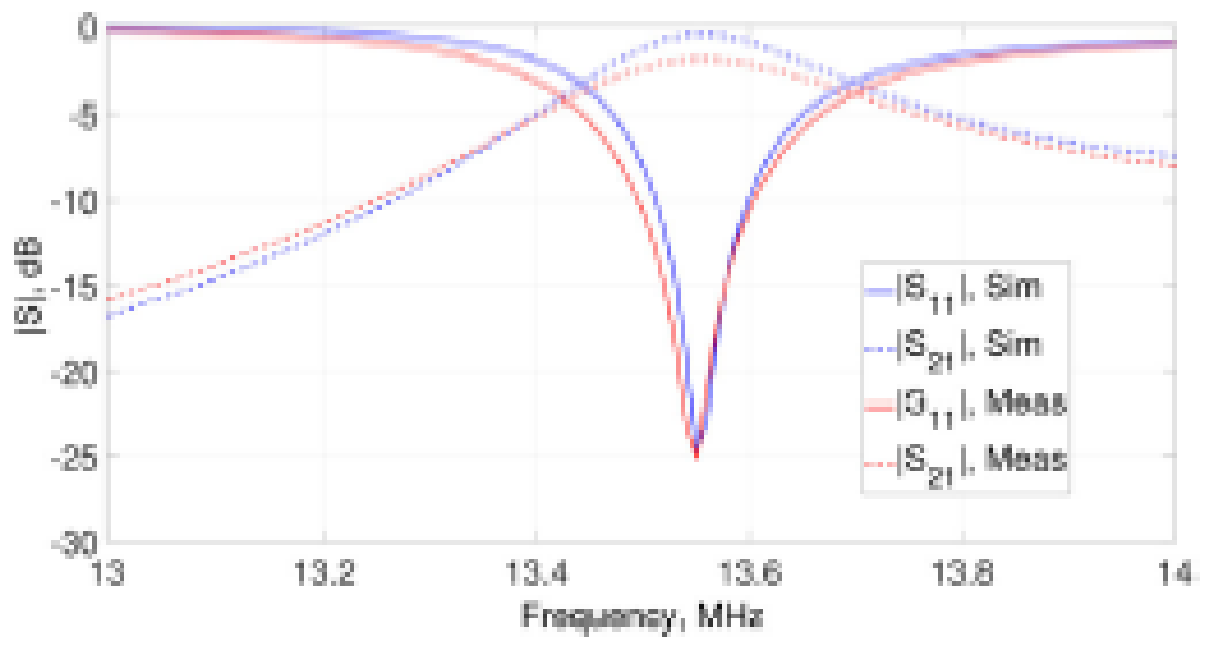

Fig. 4 Scattering parameters of the proposed WPT system inside the incubator. WPT = wireless power transfer. 
(a)

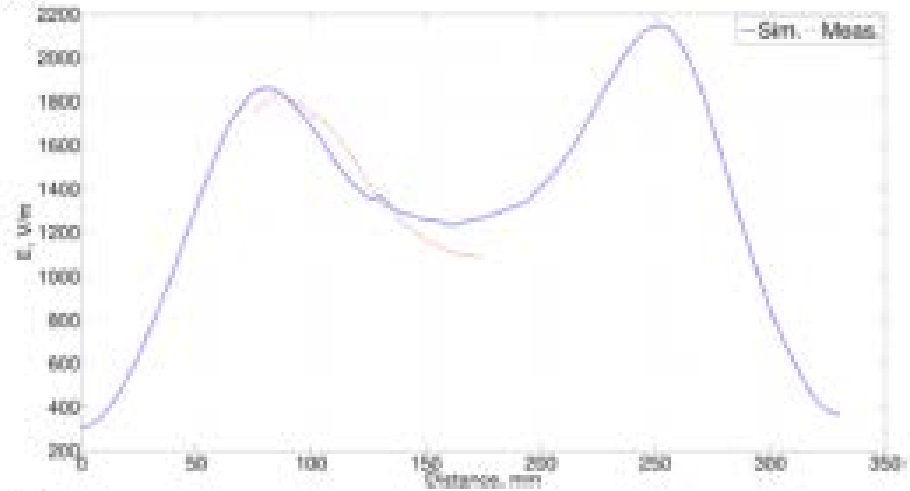

(b)

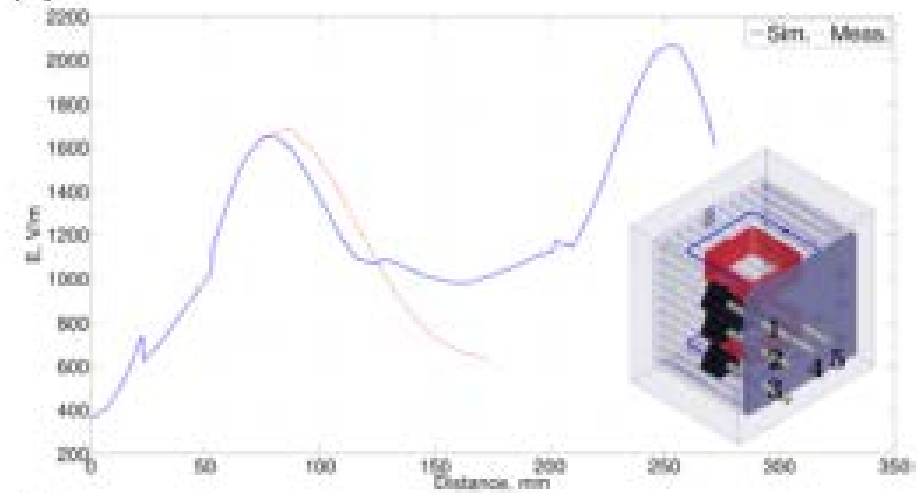

(c)

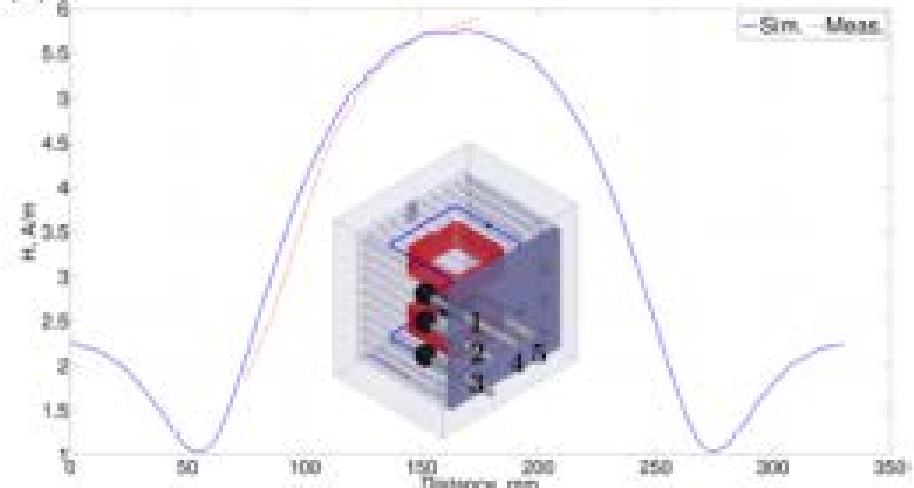

(d)

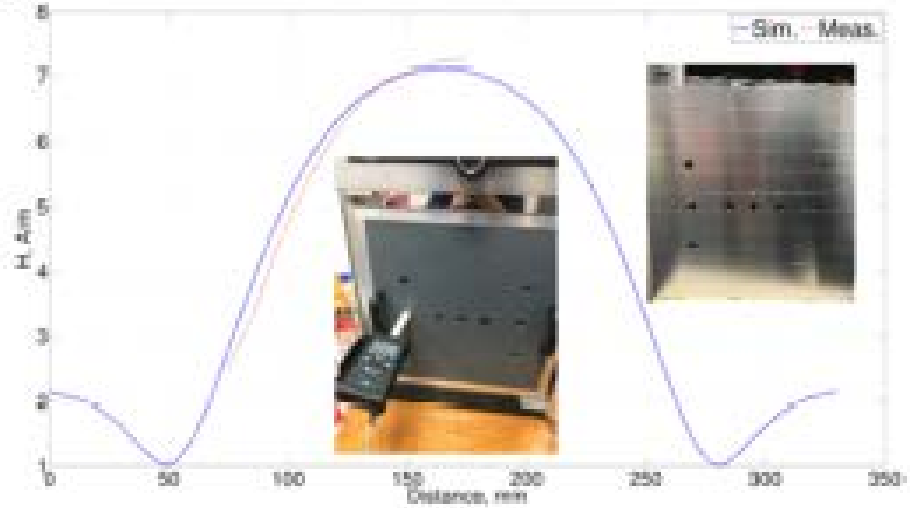

Fig. 5 Simulated and measured $\mathrm{E}$ - and $\mathrm{H}$-field distributions at $13.56 \mathrm{MHz}$ along a line passing through holes created on the incubator door. (a) E, hole \#4; (b) E, hole \#5; (c) $\mathrm{H}$, hole \#4; (d) $\mathrm{H}$, hole \#5. 

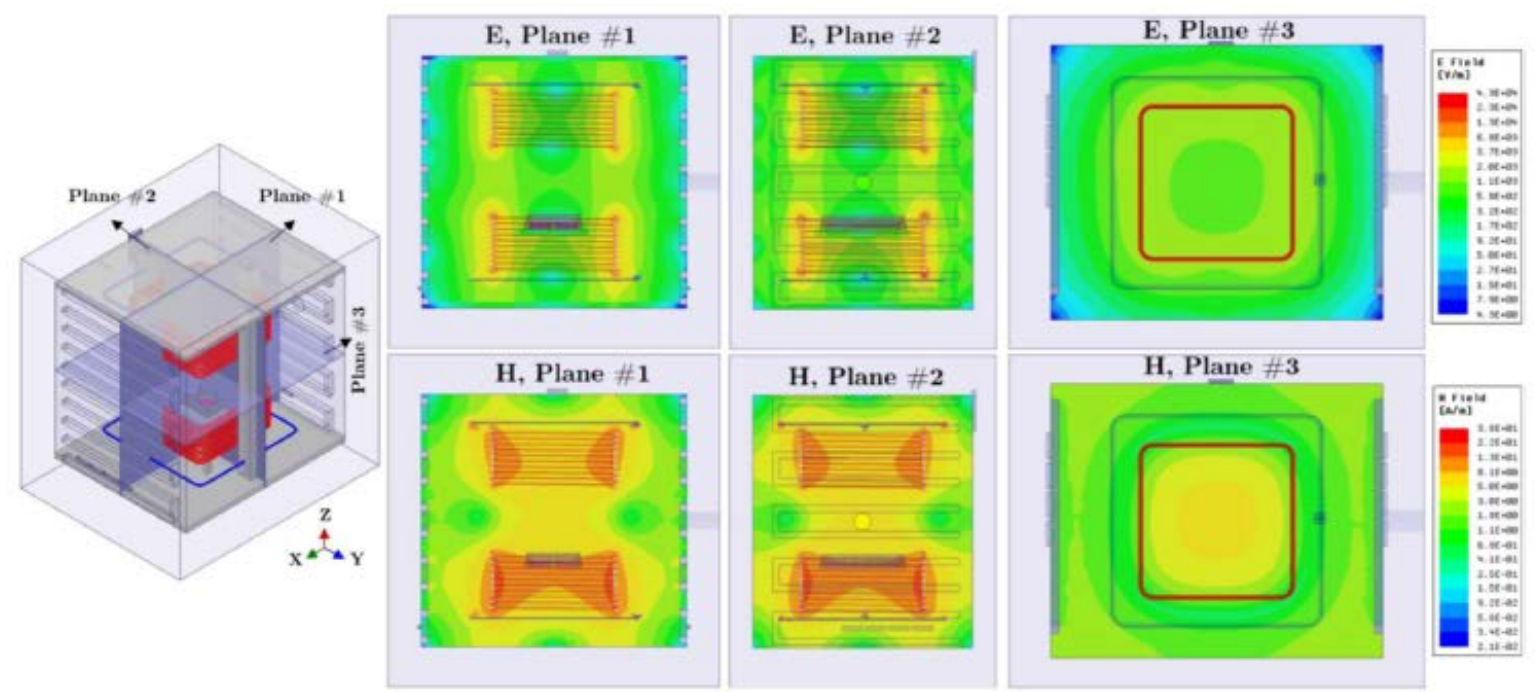

Fig. 6 E- and $\mathrm{H}$-field distributions (in log scale) inside the incubator at planes passing by the center of the $T x / R x$ for an input power of $1 \mathrm{~W}$ at $13.56 \mathrm{MHz}$. Rx=receiving; $\mathrm{Tx}=$ transmitting.
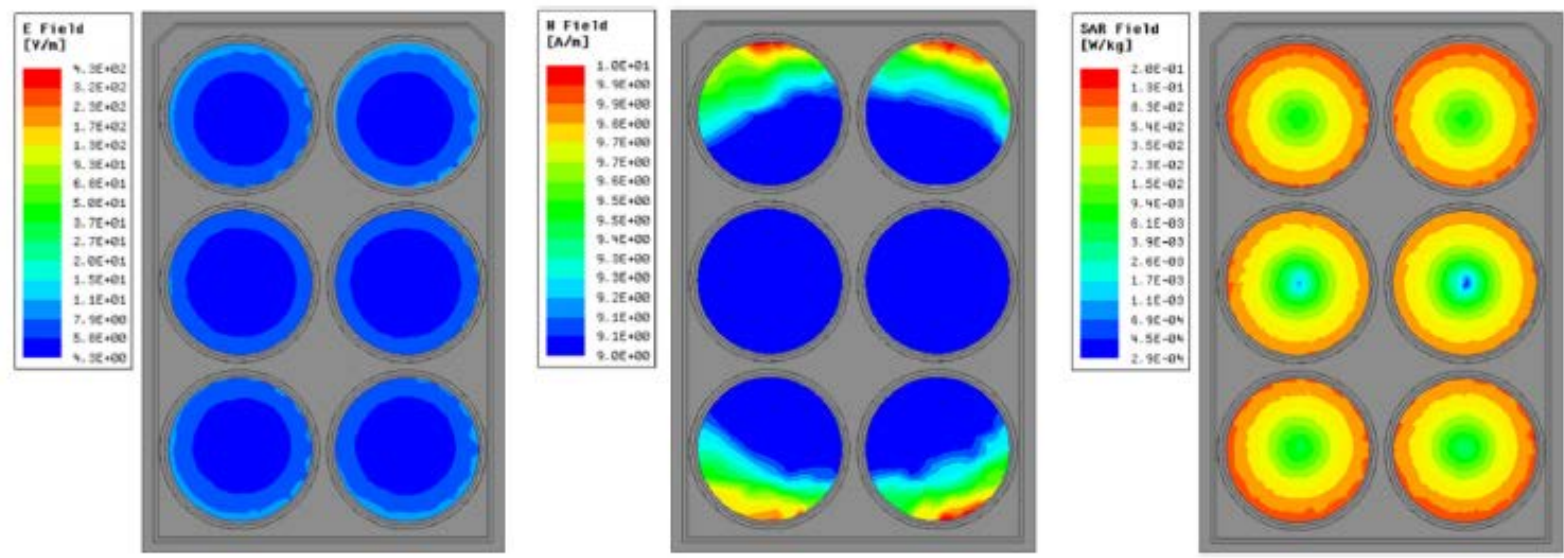

Fig. 7 E, H, and SAR distributions at the bottom of the culture medium for an input power of $1 \mathrm{~W}$ at $13.56 \mathrm{MHz}$. SAR = specific absorption rate.

\section{Tables}

TABLE 1. Design Parameters of 13.56 MHz Wireless Power Transfer System Elements With Different Geometries

\begin{tabular}{llllll}
\hline WPT system type & Loop (in cm) & Coil (in cm) & Coil turns & Pitch (in mm) & Loop-to-coil distance (in mm) \\
\hline Cylindrical & 25 (diam.) & 22 (diam.) & 10 & 8 & 12.4 \\
Planar & $30($ diam.) & 20 (inner diam.) & 8 & 4.5 & 20 \\
Square & $25 \times 25$ & $18.5 \times 18.5$ & 10 & 8 & 10 \\
\hline
\end{tabular}


TABLE 2. E, H, and SAR at Wells \#1 and \#2 for Different Configurations of WPT Systems With $d=10 \mathrm{~mm}$ and $1 \mathrm{~W}$ Input Power at $13.56 \mathrm{MHz}$

\begin{tabular}{|c|c|c|}
\hline Cyl., circular & \#1 & \#2 \\
\hline $\mathrm{E}(\mathrm{V} / \mathrm{m})$ & $5.51 \pm 1.43$ & $5.41 \pm 1.48$ \\
\hline Variation (\%) & 26.0 & 27.5 \\
\hline $\mathrm{H}(\mathrm{A} / \mathrm{m})$ & $7.45 \pm 0.018$ & $7.43 \pm 0.011$ \\
\hline Variation (\%) & 0.25 & 0.15 \\
\hline SAR (W/kg) & $0.054 \pm 0.024$ & $0.052 \pm 0.023$ \\
\hline Variation (\%) & 42.2 & 43.5 \\
\hline Annular & \#1 & \#2 \\
\hline $\mathrm{E}(\mathrm{V} / \mathrm{m})$ & $2.92 \pm 0.87$ & $2.7 \pm 0.81$ \\
\hline Variation (\%) & 29.9 & 30.3 \\
\hline $\mathrm{H}(\mathrm{A} / \mathrm{m})$ & $4.33 \pm 0.19$ & $4.02 \pm 0.05$ \\
\hline Variation (\%) & 4.5 & 1.4 \\
\hline SAR (W/kg) & $0.013 \pm 0.006$ & $0.015 \pm 0.008$ \\
\hline Variation (\%) & 50.4 & 50.7 \\
\hline Cyl., square & \#1 & \#2 \\
\hline $\mathrm{E}(\mathrm{V} / \mathrm{m})$ & $6.83 \pm 1.76$ & $6.67 \pm 2.0$ \\
\hline Variation (\%) & 25.82 & 29.97 \\
\hline $\mathrm{H}(\mathrm{A} / \mathrm{m})$ & $8.98 \pm 0.018$ & $8.91 \pm 0.06$ \\
\hline Variation (\%) & 0.20 & 0.74 \\
\hline SAR (W/kg) & $0.068 \pm 0.029$ & $0.067 \pm 0.037$ \\
\hline Variation (\%) & 42.89 & 54.81 \\
\hline
\end{tabular}


TABLE 3. E, H, and SAR at Wells \#1 and \#2 for the WPT System for Different $d$ and $1 \mathrm{~W}$ Luput Power at $1356 \mathrm{MHz}$

\begin{tabular}{|c|c|c|}
\hline$d=-20$ nn & $\# 1$ & $\# 2$ \\
\hline $\begin{array}{l}\mathrm{E}(\mathrm{W} / \mathrm{m}) \\
\text { Variation }(\%) \\
\mathrm{H}(\mathrm{A} / \mathrm{m}) \\
\text { Variation }(\%) \\
\text { SAR (W/kg) } \\
\text { Variation }(\%)\end{array}$ & $\begin{array}{l}7.98 \pm 2.16 \\
27.12 \\
10.82 \pm 0.17 \\
1.6 \\
0.094 \pm 0.042 \\
44.43\end{array}$ & $\begin{array}{l}8.72 \pm 2.43 \\
27.99 \\
11.7 \pm 0.57 \\
4.9 \\
0.11 \pm 0.058 \\
50.84\end{array}$ \\
\hline$d=-10 \mathrm{~mm}$ & $\# 1$ & $\# 2$ \\
\hline $\begin{array}{l}\mathrm{E}(\mathrm{W} / \mathrm{m}) \\
\text { Variation }(\%) \\
\mathrm{H}(\mathrm{A} / \mathrm{m}) \\
\text { Variation }(\%) \\
\text { SAR (Whg) } \\
\text { Variation (\%) }\end{array}$ & $\begin{array}{l}7.66 \pm 1.99 \\
26.01 \\
103 \pm 0.11 \\
1.06 \\
0.086 \pm 0.036 \\
42.43\end{array}$ & $\begin{array}{l}8.11 \pm 2.32 \\
28.61 \\
10.87 \pm 0.37 \\
3.43 \\
0.098 \pm 0.048 \\
49.15\end{array}$ \\
\hline$d=10 \mathrm{~mm}$ & $\# 1$ & $\# 2$ \\
\hline $\begin{array}{l}\mathrm{E}(\mathrm{V} / \mathrm{m}) \\
\text { Variation }(\%) \\
\mathrm{H}(\mathrm{A} / \mathrm{m}) \\
\text { Variation }(\%) \\
\text { SAR (W/kg) } \\
\text { Variation (\%) }\end{array}$ & $\begin{array}{l}7.31 \pm 1.83 \\
25.12 \\
9.66 \pm 0.056 \\
0.58 \\
0.078 \pm 0.032 \\
41.15\end{array}$ & $\begin{array}{l}7.46 \pm 2.12 \\
28.49 \\
9.91 \pm 0.15 \\
1.5 \\
0.089 \pm 0.04 \\
48.91\end{array}$ \\
\hline$d=+10$ min & $\# 1$ & $\# 2$ \\
\hline $\begin{array}{l}\mathrm{E}(\mathrm{V} / \mathrm{m}) \\
\text { Variation }(\%) \\
\mathrm{H}(\mathrm{A} / \mathrm{m}) \\
\text { Variation }(\%) \\
\text { SAR (W/kg) } \\
\text { Variation }(\%)\end{array}$ & $\begin{array}{l}6.54 \pm 1.94 \\
29.7 \\
8.84 \pm 0.052 \\
0.52 \\
0.064 \pm 0.092 \\
50.46\end{array}$ & $\begin{array}{l}6.77 \pm 1.7 \\
25.11 \\
8.9 \pm 0.018 \\
0.20 \\
0.067 \pm 0.08 \\
44.8\end{array}$ \\
\hline$d=+20$ nш & \#1 & $\# 2$ \\
\hline $\begin{array}{l}\mathrm{E}(\mathrm{V} / \mathrm{m}) \\
\text { Variation }(\%) \\
\mathrm{H}(\mathrm{A} / \mathrm{m}) \\
\text { Variation }(\%) \\
\text { SAR (Whg) } \\
\text { Variation }(\%)\end{array}$ & $\begin{array}{l}6.15 \pm 1.84 \\
29.97 \\
7.88 \pm 0.21 \\
2.74 \\
0.056 \pm 0.06 \\
109.47\end{array}$ & $\begin{array}{l}6.27 \pm 1.65 \\
26.39 \\
8.22 \pm 0.062 \\
0.76 \\
0.058 \pm 0.027 \\
46.36\end{array}$ \\
\hline$d=+30$ пш & $\# 1$ & $\# 2$ \\
\hline $\begin{array}{l}\mathrm{E} \text { (W/m) } \\
\text { Variation }(\%) \\
\mathrm{H}(\mathrm{A} / \mathrm{m}) \\
\text { Variation }(\%) \\
\text { SAR (W/kg) } \\
\text { Variation }(\%)\end{array}$ & $\begin{array}{l}5.78 \pm 1.9 \\
32.95 \\
7.25 \pm 0.35 \\
4.95 \\
0.05 \pm 0.062 \\
1125\end{array}$ & $\begin{array}{l}5.92 \pm 1.69 \\
27.64 \\
7.81 \pm 0.11 \\
1.42 \\
0.052 \pm 0.009 \\
48.48\end{array}$ \\
\hline
\end{tabular}


TABLE 4. E, H, and SAR at Wells \#1 and \#2 for $1 \mathrm{~W}$ Input Power at $13.56 \mathrm{MHz}$ for a Tissue Culture Plate rotated by $90^{\circ}$

\begin{tabular}{|c|c|c|}
\hline$d=+10 \mathrm{~mm}$ & \#1 & \#2 \\
\hline $\begin{array}{l}\mathrm{E}(\mathrm{V} / \mathrm{m}) \\
\text { Variation }(\%)\end{array}$ & $\begin{array}{l}6.82 \pm 1.78 \\
26.17\end{array}$ & $\begin{array}{l}6.95 \pm 1.99 \\
29.09\end{array}$ \\
\hline$d=+10 \mathrm{~mm}$ & \#1 & \#2 \\
\hline $\begin{array}{l}\mathrm{H}(\mathrm{A} / \mathrm{m}) \\
\text { Variation }(\%)\end{array}$ & $\begin{array}{l}9.04 \pm 0.018 \\
0.2\end{array}$ & $\begin{array}{l}8.95 \pm 0.006 \\
0.76\end{array}$ \\
\hline$d=+10 \mathrm{~mm}$ & $\# 1$ & \#2 \\
\hline $\begin{array}{l}\text { SAR (W/kg) } \\
\text { Variation (\%) }\end{array}$ & $\begin{array}{l}0.068 \pm 0.030 \\
43.22\end{array}$ & $\begin{array}{l}0.072 \pm 0.044 \\
60.88\end{array}$ \\
\hline
\end{tabular}

TABLE 5. Impact of the Matching Circuit, Connected to the WPT System to Shift its Resonance From 13 to $13.56 \mathrm{MHz}$, on E, H, and SAR at Wells \#1 and \#2 for 1 W Input Power

\begin{tabular}{lll}
\hline $13 \mathrm{MHz}$ & \multicolumn{1}{c}{$\# 1$} & \multicolumn{1}{c}{$\# 2$} \\
\hline $\mathrm{E}(\mathrm{V} / \mathrm{m})$ & $5.56 \pm 2.02$ & $5.65 \pm 1.71$ \\
Variation (\%) & 36.3 & 30.28 \\
$\mathrm{H}(\mathrm{A} / \mathrm{m})$ & $8.84 \pm 0.081$ & $8.94 \pm 0.025$ \\
Variation (\%) & 0.91 & 0.28 \\
SAR (W/kg) & $0.048 \pm 0.028$ & $0.047 \pm 0.023$ \\
Variation (\%) & 60.0 & 49.9 \\
\hline $13.56 \mathrm{MHz}$ & \multicolumn{1}{c}{$\# 1$} & \multicolumn{1}{c}{$\# 2$} \\
\hline E (V/m) & $2.72 \pm 0.065$ & $2.48 \pm 0.046$ \\
Variation (\%) & 48.0 & 37.21 \\
$\mathrm{H}(\mathrm{A} / \mathrm{m})$ & $4.38 \pm 0.01$ & $4.0 \pm 0.003$ \\
Variation (\%) & 4.75 & 1.77 \\
SAR (W/kg) & $0.012 \pm 2.5 \mathrm{E}-5$ & $0.0097 \pm 1.5 \mathrm{E}-5$ \\
Variation (\%) & 77.93 & 61.86 \\
\hline
\end{tabular}

TABLE 6. E, H, and SAR at Wells \#1 and \#2 for $1 \mathrm{~W}$ Input Power at $13.56 \mathrm{MHz}$ With a Half-Filled Tissue Culture Plate Medium

\begin{tabular}{lll}
\hline$d=-20 \mathrm{~mm}$ & \multicolumn{1}{c}{$\# 1$} & \multicolumn{1}{c}{$\# 2$} \\
\hline E $(\mathrm{V} / \mathrm{m})$ & $7.95 \pm 2.11$ & $8.69 \pm 2.2$ \\
Variation (\%) & 26.5 & 25.7 \\
H (A/m) & $10.87 \pm 0.17$ & $11.75 \pm 0.55$ \\
Variation (\%) & 1.5 & 4.7 \\
SAR (W/kg) & $0.093 \pm 0.040$ & $0.11 \pm 0.048$ \\
Variation (\%) & 43.8 & 43.5 \\
\hline
\end{tabular}

$\mathrm{SAR}=$ specific absorption rate. 
TABLE 7. E, H, and SAR for the Final Configuration of the Exposure System With 1 W Input Power at $13.56 \mathrm{MHz}$

\begin{tabular}{lllllll}
\hline Well number \# & \multicolumn{1}{c}{$\# 1$} & \multicolumn{1}{c}{ \#2 } & \multicolumn{1}{c}{ \#3 } & \multicolumn{1}{c}{ \#4 } & \#5 & \#6 \\
\hline E (V/m) & $7.08 \pm 1.64$ & $6.35 \pm 1.6$ & $6.95 \pm 1.72$ & $6.96 \pm 1.59$ & $6.26 \pm 1.53$ & $6.94 \pm 1.62$ \\
Variation (\%) & 23.18 & 25.18 & 24.77 & 22.87 & 24.52 & 23.7 \\
H (A/m) & $9.19 \pm 0.44$ & $8.5 \pm 0.14$ & $9.21 \pm 0.44$ & $9.04 \pm 0.45$ & $8.34 \pm 0.12$ & $9.06 \pm 0.44$ \\
Variation (\%) & 4.86 & 1.69 & 4.81 & 4.97 & 1.48 & 4.94 \\
SAR (W/kg) & $0.073 \pm 0.03$ & $0.059 \pm 0.02$ & $0.071 \pm 0.03$ & $0.071 \pm 0.028$ & $0.057 \pm 0.02$ & $0.069 \pm 0.02$ \\
Variation (\%) & 40.75 & 42.15 & 42.51 & 40.62 & 40.17 & 40.92 \\
\hline
\end{tabular}

$\mathrm{SAR}=$ specific absorption rate.

TABLE 8. MAIP to Satisfy Local SAR ICNIRP Basic

Restrictions at $13.56 \mathrm{MHz}$ for the Final Configuration of the Exposure System

\begin{tabular}{lcccccc}
\hline Well number \# & $\# 1$ & \#2 & \#3 & \#4 & \#5 & \#6 \\
\hline MAIP (W) & 27.4 & 33.9 & 28.2 & 28.2 & 35.1 & 29.0 \\
\hline
\end{tabular}

$\mathrm{MAIP}=$ maximum allowable input power SAR $=$ specific absorption rate. 